\title{
Theoretical description of the multiplicity correlations in windows separated in azimuth and rapidity
}

\author{
Vladimir Vechernin ${ }^{* \dagger}$ \\ Saint-Petersburg State University \\ E-mail: vechernin@pobox.spbu.ru
}

\begin{abstract}
The connection of the FB multiplicity correlation coefficient $b$ in windows separated in azimuth and rapidity with the two-particle correlation function $C_{2}$ is traced. Basing on this connection the general features of the dependence of the correlation coefficient $b$ on the acceptances of the observation windows and the rapidity gap between them are found a model independent way. It is demonstrated that the traditionally defined FB correlation coefficient $b$ has the strong nonlinear dependence on the acceptance of windows, going to zero with the acceptance. In this connection the suitable observables for the future FB correlation studies are proposed. It is shown a model independent way that the measurements of the FB correlations between multiplicities in two small windows separated in rapidity and azimuth enable to find the two-particle correlation function $C_{2}$ even when the particle distribution in rapidity is not flat, as in pA scattering, i.e. when the traditional di-hadron correlation analysis is not applicable.
\end{abstract}

XXII International Baldin Seminar on High Energy Physics Problems

September 15-20, 2014

JINR, Dubna, Russia

\footnotetext{
* Speaker.

${ }^{\dagger}$ The work was supported by the RFFI grant 15-02-02097-a and by the Saint-Petersburg State University grant 11.38.197.2014.
} 


\section{Introduction}

Over a long period of time, considerable attention was paid to the experimental [1]-[6] and theoretical [7]-[17] investigations of the so-called forward-backward (FB) correlation in high-energy pp and AA collisions - the correlation between multiplicities $n_{F}$ and $n_{B}$ of charged particles produced in two separated rapidity windows ("forward" and "backward"). The old problem in this correlation analysis is the separation of the so-called "volume" contribution, originating from the event-by-event fluctuation in the number of emitting sources [7].

In paper [15] it was suggested to use for this purpose the information on the event multiplicity in an additional third rapidity window, but as discussed in [16] it complicates the interpretation of obtained results. In present paper we argue that the investigation of the FB correlation between multiplicities in windows separated both in rapidity and azimuth enables to find the full two-particle correlation function $C_{2}$ including its constant component (the common "pedestal"), responsible for the long-range correlation effects [7] and containing the important physical information on the magnitude of the event-by-event fluctuation in the number of emitting sources.

The connection between the FB multiplicity correlation coefficient $b$ and the two-particle correlation function $C_{2}$ enables us a model independent way to find the general features of the dependence of the correlation coefficient $b$ on the acceptances of the observation windows. We show that the traditional definition of the FB correlation coefficient leads to its strong dependence on the acceptance of the windows, with the correlation coefficient going to zero with the acceptance.

Hence, the results obtained for the windows of different width can't be compared directly. In this connection we propose alternative suitable observables for the future FB correlation studies, which values have nonzero limit when the acceptance goes to zero.

We also compare the FB correlation approach with the so-called di-hadron correlation analysis. We see that the measurements of the FB correlation coefficient between multiplicities in two small windows separated in rapidity and azimuth enable to find the two-particle correlation function $C_{2}$ even when the particle distribution in rapidity is not flat, as e.g. in the case of pA interactions, and when the correlation function depends not only on the difference of rapidities, $\Delta \eta=\eta_{1}-\eta_{2}$, but on both of them. That is when the traditional di-hadron correlation analysis, assuming from the very beginning the dependence of two-particle correlation function only on the differences $\Delta \eta$ and $\Delta \phi$, is not applicable.

We note that even in a mid-rapidity region, where the application of the di-hadron correlation approach is justified, the results obtained by this method depend on the details of track and/or event mixing used in the approach for the imitation of the uncorrelated particle production, what leads to the uncertainties in determination of the long-range correlation component of $C_{2}$, important at the analysis of the event-by-event fluctuation of the number of sources. We show that this long-range component of $C_{2}$ can be measured unambiguously, by the FB correlation approach, which does not use any event mixture procedure, and hence avoids the associated uncertainties.

The paper is organized as follows.

In Sec. 2 we discuss the different traditional versions of the definition of the FB correlation coefficient and generalize this definition to the case of windows separated both in rapidity and azimuth. 
In Sec.3 the connection of the FB correlation coefficient with the two-particle correlation function $C_{2}$ is traced.

In Sec. 4 basing on this connection we analyze a model independent way the general features of the dependence of the correlation coefficient $b$ on the acceptances of the observation windows and the rapidity gap between them.

In Sec. 5 we propose the suitable observables for the future FB correlation studies.

The Sec.6 is devoted to the comparison of the FB correlation approach in windows separated both in rapidity and azimuth with the di-hadron correlation analysis.

Appendix A describes the calculation of integrals over rapidity and azimuth windows.

\section{Definition of the FB correlation coefficient}

Traditionally $[1,2,4,5]$ the FB correlation coefficient is defined as a coefficient $b$ in linear regression

$$
\left\langle n_{B}\right\rangle_{n_{F}}=a+b n_{F}
$$

In this case

$$
b=\frac{\left\langle n_{F} n_{B}\right\rangle-\left\langle n_{F}\right\rangle\left\langle n_{B}\right\rangle}{D_{n_{F}}},
$$

where $D_{n_{F}}$ is the variance of the multiplicity in the forward window:

$$
D_{n_{F}}=\left\langle n_{F}^{2}\right\rangle-\left\langle n_{F}\right\rangle^{2} .
$$

Clear that the value of such defined correlation coefficient changes, if one will change independently the acceptances of the forward and/or backward windows. To avoid this trivial influence one can go from $n_{F}$ and $n_{B}$ to the relative or scaled observables [20] $v_{F}=n_{F} /\left\langle n_{F}\right\rangle$ and $v_{B}=n_{B} /\left\langle n_{B}\right\rangle$. In these observables $\left\langle v_{B}\right\rangle_{v_{F}}=a_{r e l}+b_{r e l} v_{F}$ and

$$
b_{\text {rel }}=\frac{\left\langle v_{F} v_{B}\right\rangle-1}{\left\langle v_{F}^{2}\right\rangle-1}=\frac{\left\langle n_{F}\right\rangle}{\left\langle n_{B}\right\rangle} b,
$$

In some papers $[3,6]$ the following symmetrized form of (2.2) is also used,

$$
b_{s y m}=\frac{\left\langle n_{F} n_{B}\right\rangle-\left\langle n_{F}\right\rangle\left\langle n_{B}\right\rangle}{\sqrt{D_{n_{F}} D_{n_{B}}}} .
$$

For this case one can prove that $\left|b_{\text {sym }}\right| \leq 1$. Note that in the case of symmetric windows, when $\left\langle n_{F}\right\rangle=\left\langle n_{B}\right\rangle$ and $D_{n_{F}}=D_{n_{B}}$, all these definitions lead to the same result

$$
b_{r e l}=b_{s y m}=b \text {. }
$$

In present paper we study the correlation between multiplicities $n_{F}$ and $n_{B}$ in windows separated both in rapidity and in azimuth. Denote by $\delta \eta_{F}, \delta \phi_{F}$ and by $\delta \eta_{B}, \delta \phi_{B}$ the width of the forward and backward windows in rapidity and in azimuth, and by $\eta_{F}, \phi_{F}$ and $\eta_{B}, \phi_{B}$ - the positions of the centers of the windows. We'll also use the following short notation for the acceptance of forward and backward windows

$$
\delta_{F} \equiv \delta \eta_{F} \delta \phi_{F} / 2 \pi, \quad \delta_{B} \equiv \delta \eta_{B} \delta \phi_{B} / 2 \pi .
$$


By

$$
\eta_{F B} \equiv \eta_{F}-\eta_{B}, \quad \phi_{F B} \equiv \phi_{F}-\phi_{B}
$$

we denote the distance between the centers of the windows in rapidity and in azimuth.

These variables in an obvious way are connected with the so-called gaps $\eta_{\text {gap }}$ and $\phi_{\text {gap }}$ between window in rapidity and in azimuth:

$$
\eta_{F B}=\frac{\delta \eta_{F}}{2}+\eta_{g a p}+\frac{\delta \eta_{B}}{2}, \quad \phi_{F B}=\frac{\delta \phi_{F}}{2}+\phi_{g a p}+\frac{\delta \phi_{B}}{2}
$$

or for symmetric windows, when $\delta \eta_{F}=\delta \eta_{B}=\delta \eta$ and $\delta \phi_{F}=\delta \phi_{B}=\delta \phi$ :

$$
\eta_{F B}=\eta_{g a p}+\delta \eta, \quad \phi_{F B}=\phi_{g a p}+\delta \phi
$$

\section{Connection with two-particle correlation function}

One can express the FB correlation coefficient through the two-particle correlation function $C_{2}\left(\eta_{1}, \eta_{2} ; \phi_{1}, \phi_{2}\right)$. For this purpose, we introduce the $\rho_{1}(\eta, \phi)$ and $\rho_{2}\left(\eta_{1}, \phi_{1} ; \eta_{2}, \phi_{2}\right)$ - the one- and two-particle densities of charge particles:

$$
\rho_{1}(\eta, \phi)=\frac{d^{2} N}{d \eta d \phi}, \quad \rho_{2}\left(\eta_{1}, \phi_{1} ; \eta_{2}, \phi_{2}\right)=\frac{d^{4} N}{d \eta_{1} d \phi_{1} d \eta_{2} d \phi_{2}}
$$

If we integrate (3.1) over the forward acceptance interval, $\eta \in \delta \eta_{F}, \phi \in \delta \phi_{F}$, we have [21]:

$$
\begin{gathered}
\int_{\delta \eta_{F} \delta \phi_{F}} d \eta d \phi \rho_{1}(\eta, \phi)=\left\langle n_{F}\right\rangle \\
\int_{\delta \eta_{F} \delta \phi_{F}} d \eta_{1} d \phi_{1} \int_{\delta \eta_{F} \delta \phi_{F}} d \eta_{2} d \phi_{2} \rho_{2}\left(\eta_{1}, \phi_{1} ; \eta_{2}, \phi_{2}\right)=\left\langle n_{F}\left(n_{F}-1\right)\right\rangle .
\end{gathered}
$$

When we integrate over the forward, $y_{1} \in \delta \eta_{F}, \phi_{1} \in \delta \phi_{F}$, and the backward, $y_{2} \in \delta \eta_{B}, \phi_{2} \in \delta \phi_{B}$, acceptance intervals, we have

$$
\int_{\delta \eta_{F} \delta \phi_{F}} d \eta_{1} d \phi_{1} \int_{\delta \eta_{B} \delta \phi_{B}} d \eta_{2} d \phi_{2} \rho_{2}\left(\eta_{1}, \phi_{1} ; \eta_{2}, \phi_{2}\right)=\left\langle n_{F} n_{B}\right\rangle
$$

The $\left\langle n_{F}\right\rangle$ is an average multiplicity produced in the acceptance $\delta \eta_{F} \delta \phi_{F}$.

By (3.2) and (3.3) for windows of small acceptance in rapidity and azimuth we have

$$
\begin{gathered}
\rho_{1}\left(\eta_{F}, \phi_{F}\right)=\frac{\left\langle n_{F}\right\rangle}{\delta \eta_{F} \delta \phi_{F}}, \quad \rho_{2}\left(\eta_{F}, \phi_{F} ; \eta_{B}, \phi_{B}\right)=\frac{\left\langle n_{F} n_{B}\right\rangle}{\delta \eta_{F} \delta \phi_{F} \delta \eta_{B} \delta \phi_{B}} \\
\rho_{2}\left(\eta_{F}, \phi_{F} ; \eta_{F}, \phi_{F}\right)=\frac{\left\langle n_{F}\left(n_{F}-1\right)\right\rangle}{\left(\delta \eta_{F} \delta \phi_{F}\right)^{2}}
\end{gathered}
$$

These formulae enable the experimental measurement of the one- and two-particle densities of charge particles $\rho_{1}(\eta, \phi)$ and $\rho_{2}\left(\eta_{1}, \phi_{1} ; \eta_{2}, \phi_{2}\right)$, and hence the two-particle correlation function $C_{2}$, which is introduced in a standard way:

$$
C_{2}\left(\eta_{1}, \eta_{2} ; \phi_{1}, \phi_{2}\right)=\frac{\rho_{2}\left(\eta_{1}, \eta_{2} ; \phi_{1}, \phi_{2}\right)}{\rho_{1}\left(\eta_{1}, \phi_{1}\right) \rho_{1}\left(\eta_{1}, \phi_{2}\right)}-1
$$


Substituting (3.4) in (3.6) we get for windows of small acceptance in rapidity and azimuth:

$$
C_{2}\left(\eta_{F}, \phi_{F} ; \eta_{B}, \phi_{B}\right)=\frac{\left\langle n_{F} n_{B}\right\rangle-\left\langle n_{F}\right\rangle\left\langle n_{B}\right\rangle}{\left\langle n_{F}\right\rangle\left\langle n_{B}\right\rangle}=\left\langle\frac{n_{F}}{\left\langle n_{F}\right\rangle} \frac{n_{B}}{\left\langle n_{B}\right\rangle}\right\rangle-1
$$

Important that by (3.7) the observation of multiplicity-multiplicity correlation with two small (in azimuth and rapidity) windows, enables to measure the two-particle correlation function $C_{2}$ in accordance with the standard definition (3.6) even in the absence of the translation invariance in rapidity and without using an event mixing procedure, usually applied in a di-hadron correlation analysis (see the discussion in Section 6). Like this it is also valid for nonhomogeneous distributions, which take place for non-symmetric reaction, as pA scattering, and for symmetric reaction at large rapidity gaps.

Note also that if in formula (3.7) we'll mean by $n_{F}$ and $n_{B}$ the multiplicities of particles with the certain transverse momenta belonging correspondingly to the intervals $\delta p_{\mathrm{T} F}$ and $\delta p_{\mathrm{T} B}$, than by (3.7) one can measure the two-particle correlation function $C_{2}$ between particles belonging to the different transverse momentum intervals, like in the so-called triggered di-hadron correlation approach. In principle, this enables using the small $\delta p_{\mathrm{T} F}$ and $\delta p_{\mathrm{T} B}$ intervals to measure the whole two-particle correlation function $C_{2}\left(\mathbf{p}_{F}, \mathbf{p}_{B}\right)$ with the 3-momenta $\mathbf{p}_{F}$ and $\mathbf{p}_{B}$ being the centers of the $\delta \eta_{F} \delta \phi_{F} \delta p_{\mathrm{T} F}$ and $\delta \eta_{B} \delta \phi_{B} \delta p_{\mathrm{T} B}$ intervals.

In general case the azimuth rotation invariance leads to some simplifications:

$$
\rho_{1}(\eta, \phi)=\rho_{1}(\eta) / 2 \pi, \quad \rho_{2}\left(\eta_{1}, \phi_{1} ; \eta_{2}, \phi_{2}\right)=\rho_{2}\left(\eta_{1}, \eta_{2} ; \phi_{1}-\phi_{2}\right) /(2 \pi)^{2}
$$

and

$$
C_{2}\left(\eta_{1}, \eta_{2} ; \phi_{1}-\phi_{2}\right)=\frac{\rho_{2}\left(\eta_{1}, \eta_{2} ; \phi_{1}-\phi_{2}\right)}{\rho_{1}\left(\eta_{1}\right) \rho_{1}\left(\eta_{2}\right)}-1
$$

In the case of windows of arbitrary widths by (3.2) and (3.3) we have

$$
\begin{gathered}
\left\langle n_{F} n_{B}\right\rangle-\left\langle n_{F}\right\rangle\left\langle n_{B}\right\rangle=\left\langle n_{F}\right\rangle\left\langle n_{B}\right\rangle I_{F B}, \\
D_{n_{F}}=\left\langle n_{F}\right\rangle+\left\langle n_{F}\right\rangle^{2} I_{F F},
\end{gathered}
$$

where

$$
\begin{gathered}
\left\langle n_{F}\right\rangle=\frac{\delta \phi_{F}}{2 \pi} \int_{\delta \eta_{F}} d \eta \rho_{1}(\eta) \\
I_{F B}=\frac{1}{(2 \pi)^{2}\left\langle n_{F}\right\rangle\left\langle n_{B}\right\rangle} \int_{\delta \eta_{F} \delta \phi_{F}} d \eta_{1} d \phi_{1} \int_{\delta \eta_{B} \delta \phi_{B}} d \eta_{2} d \phi_{2} \rho_{1}\left(\eta_{1}\right) \rho_{1}\left(\eta_{2}\right) C_{2}\left(\eta_{1}, \eta_{2} ; \phi_{1}-\phi_{2}\right), \\
I_{F F}=\frac{1}{(2 \pi)^{2}\left\langle n_{F}\right\rangle^{2}} \int_{\delta \eta_{F} \delta \phi_{F}} d \eta_{1} d \phi_{1} \int_{\delta \eta_{F} \delta \phi_{F}} d \eta_{2} d \phi_{2} \rho_{1}\left(\eta_{1}\right) \rho_{1}\left(\eta_{2}\right) C_{2}\left(\eta_{1}, \eta_{2} ; \phi_{1}-\phi_{2}\right) .
\end{gathered}
$$

What gives for the correlation coefficient

$$
b_{\text {rel }}=\frac{\left\langle n_{F}\right\rangle}{\left\langle n_{B}\right\rangle} b=\frac{\left\langle n_{F}\right\rangle I_{F B}}{1+\left\langle n_{F}\right\rangle I_{F F}} .
$$

Important that in the absence of correlation, when $C_{2}=0$, we have $I_{F B}=I_{F F}=0$ and simultaneously the poissonian multiplicity distribution in the forward window with $D_{n_{F}}=\left\langle n_{F}\right\rangle$, (3.11). 
Further simplification of the integrals (3.13) and (3.14) is discussed in Appendix A. Note only that, for example, in the important case of FB windows separated only in rapidity (i.e. when $\left.\delta \phi_{F}=\delta \phi_{B}=2 \pi\right)$, by (7.5) we have

$$
\begin{gathered}
\left\langle n_{F}\right\rangle=\int_{\delta \eta_{F}} d \eta \rho_{1}(\eta) \\
I_{F B}=\frac{1}{\left\langle n_{F}\right\rangle\left\langle n_{B}\right\rangle} \int_{\delta \eta_{B}} d \eta_{1} \int_{\delta \eta_{F}} d \eta_{2} \rho_{1}\left(\eta_{1}\right) \rho_{1}\left(\eta_{2}\right) C_{2}\left(\eta_{1}, \eta_{2}\right), \\
I_{F F}=\frac{1}{\left\langle n_{F}\right\rangle^{2}} \int_{\delta \eta_{F}} d \eta_{1} \int_{\delta \eta_{F}} d \eta_{2} \rho_{1}\left(\eta_{1}\right) \rho_{1}\left(\eta_{2}\right) C_{2}\left(\eta_{1}, \eta_{2}\right),
\end{gathered}
$$

where

$$
C_{2}\left(\eta_{1}, \eta_{2}\right)=\frac{1}{\pi} \int_{0}^{\pi} d \phi C_{2}\left(\eta_{1}, \eta_{2} ; \phi\right)
$$

For windows, which are small both in rapidity and in azimuth (within which one can consider the $C_{2}\left(\eta_{1}, \eta_{2} ; \phi_{1}-\phi_{2}\right)$ and $\rho_{1}(\eta)$ to be constant) we have

$$
\begin{gathered}
\left\langle n_{F}\right\rangle=\rho_{1}\left(\eta_{F}\right) \delta_{F}, \quad\left\langle n_{B}\right\rangle=\rho_{1}\left(\eta_{B}\right) \delta_{B}, \\
I_{F B}=C_{2}\left(\eta_{F}, \eta_{B} ; \phi_{F B}\right), \\
I_{F F}=C_{2}\left(\eta_{F}, \eta_{F} ; 0\right), \\
D_{n_{F}}=\left\langle n_{F}\right\rangle\left[1+\left\langle n_{F}\right\rangle C_{2}\left(\eta_{F}, \eta_{F} ; 0\right)\right],
\end{gathered}
$$

and

$$
b_{r e l}=\frac{\left\langle n_{F}\right\rangle}{\left\langle n_{B}\right\rangle} b=\frac{\left\langle n_{F}\right\rangle C_{2}\left(\eta_{F}, \eta_{B} ; \phi_{F B}\right)}{1+\left\langle n_{F}\right\rangle C_{2}\left(\eta_{F}, \eta_{F} ; 0\right)} .
$$

Remember our short notations (2.7) and (2.8). We see that the correlation coefficient (2.4), even defined in scaled variables, still depends through $\left\langle n_{F}\right\rangle$ on the acceptance $\delta_{F}$ of the forward window, that was observed earlier $[18,19]$ in a framework of a simple model.

In the case when both small FB windows are situated in the central region, where one can suppose the translation invariance in rapidity:

$$
\rho_{1}(\eta)=\rho_{0}, \quad C_{2}\left(\eta_{1}, \eta_{2} ; \phi\right)=C_{2}\left(\eta_{1}-y_{2} ; \phi\right)
$$

the formulae (3.20)-(3.24) admit further simplification:

$$
\begin{gathered}
\left\langle n_{F}\right\rangle=\rho_{0} \delta_{F}, \quad\left\langle n_{B}\right\rangle=\rho_{0} \delta_{B}, \\
D_{n_{F}}=\left\langle n_{F}\right\rangle\left[1+\delta_{F} \rho_{0} C_{2}(0,0)\right], \\
b_{r e l}=\frac{\delta_{F}}{\delta_{B}} b=\frac{\delta_{F} \rho_{0} C_{2}\left(\eta_{F B}, \phi_{F B}\right)}{1+\delta_{F} \rho_{0} C_{2}(0,0)} .
\end{gathered}
$$

At last for large windows situated in the central rapidity region along with (3.25) and (3.26) we must to use the formulae (3.11) and (3.15), with the following expressions for $I_{F B}$ and $I_{F F}$ :

$$
\begin{gathered}
I_{F B}=\left(\delta \eta_{F} \delta \phi_{F} \delta \eta_{B} \delta \phi_{B}\right)^{-1} \int_{\delta \eta_{F} \delta \phi_{F}} d \eta_{1} d \phi_{1} \int_{\delta \eta_{B} \delta \phi_{B}} d \eta_{2} d \phi_{2} C_{2}\left(\eta_{1}-\eta_{2} ; \phi_{1}-\phi_{2}\right) \\
I_{F F}=\left(\delta \eta_{F} \delta \phi_{F}\right)^{-2} \int_{\delta \eta_{F} \delta \phi_{F}} d \eta_{1} d \phi_{1} \int_{\delta \eta_{F} \delta \phi_{F}} d \eta_{2} d \phi_{2} C_{2}\left(\eta_{1}-\eta_{2} ; \phi_{1}-\phi_{2}\right)
\end{gathered}
$$

(further simplification of the integrals see in Appendix A). 


\section{FB correlation coefficient dependence on the window acceptances}

The connection, (3.15), between the correlation coefficient $b$ and the two-particle correlation function $C_{2}$, discussed in previous section, enables a model-independent way to find the general features of the dependence of the correlation coefficient $b$ on the acceptance of the observation windows, mentioned above after the formula (3.24). For simplicity we will trace this dependence in the case of FB windows separated only in rapidity, i.e. when $\delta \phi_{F}=\delta \phi_{B}=2 \pi$, see formulae (3.15)-(3.19).

As a first step, using these formulae and the general relation (3.11), we find the following expression for the multiplicity variance in the forward window $D_{n_{F}}$ through the two-particle correlation function $C_{2}\left(\eta_{1}, \eta_{2}\right)$ :

$$
D_{n_{F}}=\left\langle n_{F}\right\rangle+\int_{\delta \eta_{F}} d \eta_{1} \int_{\delta \eta_{F}} d \eta_{2} \rho_{1}\left(\eta_{1}\right) \rho_{1}\left(\eta_{2}\right) C_{2}\left(\eta_{1}, \eta_{2}\right)
$$

In this formula the both integrations on $\eta_{1}$ and $\eta_{2}$ are performed over the forward window. The expression (4.1) is exact and model independent. It confirms the well-known fact [7, 21], that the multiplicity fluctuations in a given window are driven by intrinsic 2-particle correlations. Really, by this expression we see that the two-particle correlation function $C_{2}\left(\eta_{1}, \eta_{2}\right)$ determines the deviation of $D_{n_{F}}$ from $\left\langle n_{F}\right\rangle$.

In the absence of correlations, when $\rho_{2}\left(\eta_{1}, \eta_{2}\right)=\rho_{1}\left(\eta_{1}\right) \rho_{1}\left(\eta_{2}\right)$ and $C_{2}\left(\eta_{1}, \eta_{2}\right)=0$, we have the poissonian distribution for the multiplicity in any window, $D_{n_{F}}=\left\langle n_{F}\right\rangle$. To prove rigorously this statement one has to consider the expressions for higher particle distributions similar to the expression (3.2):

$$
\int_{\delta \eta_{F}} d \eta_{1} \int_{\delta \eta_{F}} d \eta_{2} \int_{\delta \eta_{F}} d \eta_{3} \rho_{3}\left(\eta_{1}, \eta_{2}, \eta_{3}\right)=\left\langle n_{F}\left(n_{F}-1\right)\left(n_{F}-2\right)\right\rangle
$$

and so on. Then in the absence of correlations, when $\rho_{3}\left(\eta_{1}, \eta_{2}, \eta_{3}\right)=\rho_{1}\left(\eta_{1}\right) \rho_{1}\left(\eta_{2}\right) \rho_{1}\left(\eta_{3}\right)$ and so on, one gets the poissonian relations between all moments of the multiplicity distribution.

In general case, using the relations (3.15)-(3.19), we have the following exact and model independent presentation for the FB correlation coefficient $b$ through the two-particle correlation function $C_{2}$ :

$$
b=\frac{\int_{\delta \eta_{F}} d \eta_{1} \int_{\delta \eta_{B}} d \eta_{2} \rho_{1}\left(\eta_{1}\right) \rho_{1}\left(\eta_{2}\right) C_{2}\left(\eta_{1}, \eta_{2}\right)}{\left\langle n_{F}\right\rangle+\int_{\delta \eta_{F}} d \eta_{1} \int_{\delta \eta_{F}} d \eta_{2} \rho_{1}\left(\eta_{1}\right) \rho_{1}\left(\eta_{2}\right) C_{2}\left(\eta_{1}, \eta_{2}\right)}
$$

(compare with formula (34) in [7]). Note that in the numerator the $\eta_{1}$ integration is performed over the forward window and $\eta_{2}$ over the backward window in contrast with the denominator, where both integrations are performed over the forward window.

In central rapidity region we can simplify the expressions (4.1) and (4.2) using that in this case, as defined in (3.25) and (3.26), the $\rho_{1}(\eta)=\left\langle n_{F}\right\rangle / \delta \eta_{F}=\left\langle n_{B}\right\rangle / \delta \eta_{B}=\rho_{0}$ is constant and the two-particle correlation function $C_{2}\left(\eta_{1}, \eta_{2}\right)$ depends only on the difference $\Delta \eta \equiv \eta_{1}-\eta_{2}$, $C_{2}\left(\eta_{1}, \eta_{2}\right)=C_{2}(\Delta \eta)$. In this case for $b_{r e l}$ we have

$$
b_{r e l}=\frac{\delta \eta_{F}}{\delta \eta_{B}} b=\frac{\frac{\left\langle n_{F}\right\rangle}{\delta \eta_{F} \delta \eta_{B}} \int_{\delta \eta_{F}} d \eta_{1} \int_{\delta \eta_{B}} d \eta_{2} C_{2}\left(\eta_{1}-\eta_{2}\right)}{1+\frac{\left\langle n_{F}\right\rangle}{\delta \eta_{F}^{2}} \int_{\delta \eta_{F}} d \eta_{1} \int_{\delta \eta_{F}} d \eta_{2} C_{2}\left(\eta_{1}-\eta_{2}\right)},
$$


at that

$$
D_{n_{F}}=\left\langle n_{F}\right\rangle+\frac{\left\langle n_{F}\right\rangle^{2}}{\delta \eta_{F}^{2}} \int_{\delta \eta_{F}} d \eta_{1} \int_{\delta \eta_{F}} d \eta_{2} C_{2}\left(\eta_{1}-\eta_{2}\right)
$$

Important, that as shown in [7] the two-particle correlation function $C_{2}$, along with the local in rapidity short-range (SR) contribution (arising due to details of a single string decay, decay of resonances and so on) contains also the constant (independent on $\eta_{1}$ and $\eta_{2}$ ) long-range (LR) contribution - the "pedestal", arising due to fluctuation in the number of cut pomerons, or more generally due to fluctuation in the number and characteristics of emitting sources (strings). So we can write

$$
C_{2}(\Delta \eta)=C_{2}^{S R}(\Delta \eta)+C_{2}^{L R}
$$

The SR contribution is local in rapidity, so at large $\Delta \eta$ we have $C_{2}^{S R}(\Delta \eta) \rightarrow 0$ and $C_{2}(\Delta \eta) \rightarrow C_{2}^{L R}$. The LR contribution was used in paper [7] to evaluate the event-by-event fluctuation in number of cut pomerons, $n$, through the relation $C_{2}^{L R}=\left\langle n^{2}\right\rangle /\langle n\rangle^{2}-1$. It was found that these fluctuations are far from poissonian especially in the case of AA scattering [26].

Note that the integrals in the formulae (4.3) and (4.4) can be simplified using that their integrands depend only on the difference $\Delta \eta \equiv \eta_{1}-\eta_{2}$ due to the translation invariance. Integrating over $\eta_{1}+\eta_{2}$ and substituting (4.5) in (4.1) we can find, for example, the SR and LR contributions into the forward window multiplicity variance:

$$
D_{n_{F}}=\left\langle n_{F}\right\rangle+\frac{2\left\langle n_{F}\right\rangle^{2}}{\delta \eta_{F}^{2}} \int_{0}^{\delta \eta_{F}} d \eta C_{2}^{S R}(\eta)\left(\delta \eta_{F}-\eta\right)+\left\langle n_{F}\right\rangle^{2} C_{2}^{L R}
$$

see Appendix A for details.

The two-particle correlation function $C_{2}(\Delta \eta),(4.5)$, has the maximum at $\Delta \eta=0$ with value

$$
C_{2}(0)=C_{2}^{S R}(0)+C_{2}^{L R}
$$

and decreases to $C_{2}^{L R}$, when the $\Delta \eta$ is larger, then the typical correlation length $\eta_{0}$, which is of order of one rapidity unit:

$$
C_{2}(\Delta \eta) \rightarrow C_{2}^{L R} \quad \text { at } \quad \Delta \eta>\eta_{0}
$$

Taking into account these very general considerations we find that for the neighbour and small enough windows, when all distances $\eta_{1}-\eta_{2}$ entering the integrals in the numerator and denominator of (4.3) is smaller then the typical correlation length $\eta_{0}, \Delta \eta<\eta_{0}$, we can use the following evaluation for these integrals:

$\int_{\delta \eta_{F}} d \eta_{1} \int_{\delta \eta_{B}} d \eta_{2} C_{2}\left(\eta_{1}-\eta_{2}\right) \approx \delta \eta_{F} \delta \eta_{B} C_{2}(0), \quad \int_{\delta \eta_{F}} d \eta_{1} \int_{\delta \eta_{F}} d \eta_{2} C_{2}\left(\eta_{1}-\eta_{2}\right) \approx\left(\delta \eta_{F}\right)^{2} C_{2}(0)$

Restricting by the case of symmetric windows, $\delta \eta_{F}=\delta \eta_{B}$, we find the following evaluation for the correlation coefficient $b$ for the neighbour and small enough windows:

$$
b^{\text {small gap }} \approx \frac{\left\langle n_{F}\right\rangle C_{2}(0)}{1+\left\langle n_{F}\right\rangle C_{2}(0)}=\frac{\delta \eta_{F} a}{1+\delta \eta_{F} a}
$$


at that

$$
D_{n_{F}}=\left\langle n_{F}\right\rangle+\left\langle n_{F}\right\rangle^{2} C_{2}(0)=\left\langle n_{F}\right\rangle\left(1+\delta \eta_{F} a\right),
$$

with $a=\rho_{0} C_{2}(0)$, where $\rho_{0}$ is defined in (3.25) and (3.26).

So we see a model-independent way that the dependence on the width of observation windows in the case with small gap between them is described by one parametric formula (4.10). By (4.7) we see that $C_{2}(0)$ and hence the parameter $a$ in (4.10) includes the contribution from all (short- and long-range) correlations. We would like also to emphasize that at small gaps the relation (4.10) will fulfilled in any model. So the experimental observation of the dependence on $\delta \eta_{F}$ of this type at small gaps can not be considered as an argument in favour of some specific model.

The other important case is a configuration with two small observation windows, $\delta \eta_{F}=\delta \eta_{B}<$ $\eta_{0}$, but with a large gap between them, i.e. with a gap which is larger then the correlation length $\eta_{0}$. In this case the typical distances $\eta_{1}-\eta_{2}$, entering the integral in the numerator of (4.3), will be also larger then the correlation length $\eta_{0}$, and by (4.8) we can use the following evaluation for this integral:

$$
\int_{\delta \eta_{F}} d \eta_{1} \int_{\delta \eta_{B}} d \eta_{2} C_{2}\left(\eta_{1}-\eta_{2}\right) \approx \delta \eta_{F} \delta \eta_{B} C_{2}^{L R}
$$

Whereas the evaluation for the integral in the denominator of (4.3), i.e. for the $n_{F}$ variance, remains the same, see formulae (4.9) and (4.11). So we find the following evaluation for the correlation coefficient $b$ for small windows separated by a large rapidity gap:

$$
b^{\text {large gap }} \approx \frac{\left\langle n_{F}\right\rangle C_{2}^{L R}}{1+\left\langle n_{F}\right\rangle C_{2}(0)}=\frac{\delta \eta_{F} a^{\prime}}{1+\delta \eta_{F} a}
$$

with $a^{\prime}=\rho_{0} C_{2}^{L R}$. In this case we see the same type of the dependence of the correlation coefficient $b$ on the width of observation windows, as in (4.10), but with the smaller constant $a^{\prime}$ in the numerator of (4.13), because by (4.7) $C_{2}(0)=C_{2}^{S R}(0)+C_{2}^{L R}>C_{2}^{L R}$ and hence $a>a^{\prime}$. By (4.10) and (4.13) we find for the ratio of the correlation coefficients:

$$
\frac{b^{\text {large gap }}}{b^{\text {small gap }}}=\frac{a^{\prime}}{a}=\frac{C_{2}^{L R}}{C_{2}(0)}=\frac{C_{2}^{L R}}{C_{2}^{S R}+C_{2}^{L R}}<1 .
$$

These general dependencies of the correlation coefficient $b$ on the width of observation windows and on the value of the gap between them are experimentally observed in the preliminary ALICE data on FB correlations in pp collisions at 0.9, 2.76 and $7 \mathrm{TeV}$ at LHC, see e.g. Figs.5 and 6 in [27].

\section{Alternative observables}

On the base of above analysis we can discuss the introduction of more suitable observables for the future FB correlation studies.

From the equations (3.20)-(3.24) we see that if the acceptance of small symmetric FB windows goes to zero, $\delta_{F}=\delta_{B} \rightarrow 0$, then all traditionally defined FB correlation coefficients $b(2.2), b_{r e l}$ (2.4) and $b_{\text {sym }}$ (2.5) also go to zero. Recall that in this case $b=b_{\text {rel }}=b_{\text {sym }}$ (2.6). This unpleasant dependence of the correlation coefficients on the width of the windows arises due to behavior of 
the variance $D_{n_{F}}$ in the denominator of (2.2). Really by (3.10) and (3.20)-(3.23) we see a modelindependent way that in this limit $\left\langle n_{F} n_{B}\right\rangle-\left\langle n_{F}\right\rangle\left\langle n_{B}\right\rangle \sim \delta_{F} \delta_{B}$ and $D_{n_{F}} \sim \delta_{F}$. We can rid of this drawback if in the definition (2.2) we normalize the correlator $\left\langle n_{F} n_{B}\right\rangle-\left\langle n_{F}\right\rangle\left\langle n_{B}\right\rangle$ by the product $\left\langle n_{F}\right\rangle\left\langle n_{B}\right\rangle$ instead of the $D_{n_{F}}$ and introduce, as an observable, the modified correlation coefficient:

$$
\beta_{\text {mod }} \equiv \frac{\left\langle n_{F} n_{B}\right\rangle-\left\langle n_{F}\right\rangle\left\langle n_{B}\right\rangle}{\left\langle n_{F}\right\rangle\left\langle n_{B}\right\rangle}=\left\langle\frac{n_{F}}{\left\langle n_{F}\right\rangle} \frac{n_{B}}{\left\langle n_{B}\right\rangle}\right\rangle-1
$$

Then for windows, which are small both in rapidity and in azimuth, by (3.21) we have

$$
\beta_{\text {mod }}=C_{2}\left(\eta_{F}, \eta_{B} ; \phi_{F B}\right)
$$

In the case of the FB windows, which are small only in rapidity and large $\left(\delta \phi_{F}=\delta \phi_{B}=2 \pi\right)$ in azimuth

$$
\beta_{\text {mod }}=C_{2}\left(\eta_{F}, \eta_{B}\right)=\frac{1}{\pi} \int_{0}^{\pi} d \phi C_{2}\left(\eta_{F}, \eta_{B} ; \phi\right)
$$

where we have take into account (3.16)-(3.19). We see that at small acceptances of windows the $\beta_{\text {mod }},(5.1)$, has the two-particle correlation function, as the finite limit at $\delta_{F}=\delta_{B} \rightarrow 0$, in contrast to $b, b_{\text {rel }}$ and $b_{\text {sym }}$.

Note that the traditionally defined (2.2) correlation coefficient $b$, used above, is also proportional to the two-particle correlation function $C_{2}\left(\eta_{F}, \eta_{B} ; \phi_{F B}\right)$ (see (3.24)), but the proportionality factor depends on the width of windows and goes to zero at $\delta_{F}=\delta_{B} \rightarrow 0$.

Another possibility, which follows from (3.23), is to use for the normalization in (2.5) the differences $D_{n_{F}}-\left\langle n_{F}\right\rangle$ and $D_{n_{B}}-\left\langle n_{B}\right\rangle$ instead of $D_{n_{F}}$ and $D_{n_{B}}$ and to introduce another correlation observable, defined as follows:

$$
\beta_{r o b} \equiv \frac{\left\langle n_{F} n_{B}\right\rangle-\left\langle n_{F}\right\rangle\left\langle n_{B}\right\rangle}{\sqrt{D_{n_{F}}-\left\langle n_{F}\right\rangle} \sqrt{D_{n_{B}}-\left\langle n_{B}\right\rangle}} .
$$

Then again for windows, which are small both in rapidity and in azimuth, by (3.21) and (3.23) we have

$$
\beta_{r o b}=\frac{C_{2}\left(\eta_{F}, \eta_{B} ; \phi_{F B}\right)}{\sqrt{C_{2}\left(\eta_{F}, \eta_{F} ; 0\right) C_{2}\left(\eta_{B}, \eta_{B} ; 0\right)}} .
$$

For small windows in mid-rapidity region it reduces to

$$
\beta_{r o b}=\frac{C_{2}\left(\eta_{F B}, \phi_{F B}\right)}{C_{2}(0,0)}
$$

In the case when the FB windows are $\delta \phi_{F}=\delta \phi_{B}=2 \pi$ in azimuth and small in rapidity, we'll have

$$
\beta_{r o b}=\frac{C_{2}\left(\eta_{F}, \eta_{B}\right)}{\sqrt{C_{2}\left(\eta_{F}, \eta_{F}\right) C_{2}\left(\eta_{B}, \eta_{B}\right)}}
$$

where $C_{2}\left(\eta_{F}, \eta_{B}\right)$ is defined by (3.19) and

$$
\beta_{r o b}=\frac{C_{2}\left(\eta_{F B}\right)}{C_{2}(0)}
$$


at central region, where the translation invariance in rapidity holds. We see that the $\beta_{\text {rob }}$, as the $\beta_{\text {mod }}$, has a finite limit at small acceptances of windows in contrast to the traditionally used $b, b_{r e l}$ and $b_{\text {sym }}$.

Note that the second definition (5.4) is closely connected with so-called robust variance [21, 22, 23]:

$$
R_{n}=\frac{D_{n}-\langle n\rangle}{\langle n\rangle^{2}}
$$

By (5.1) and (5.4) we have

$$
\beta_{\text {rob }}=\frac{\beta_{\text {mod }}}{\sqrt{R_{n_{F}} R_{n_{B}}}} .
$$

So comparing the different definitions of the multiplicity correlation coefficient, we see that the traditional definitions (2.2), (2.4) and (2.5) of the FB correlation coefficient lead to its strong dependence on the acceptance of windows, with the correlation coefficient going to zero with the window width. Hence, the results obtained for windows of different width can't be compared directly. In this connection it can be suitable to use in the future FB correlation studies the proposed observables (5.1) and (5.4), which values have nonzero limits when the acceptance of windows goes to zero.

\section{Comparison with the untriggered di-hadron correlation approach}

In practice, in di-hadron correlation analysis, the following alternative definition of the twoparticle correlation function $C$ is in use [24, 25]:

$$
C=S / B-1,
$$

where

$$
S=\frac{d^{2} N}{d \Delta \eta d \Delta \phi} .
$$

Here $\Delta \eta=\eta_{1}-\eta_{2}$ and $\Delta \phi=\phi_{1}-\phi_{2}$ are the distances between two particles in rapidity and in azimuth, and one takes into account all possible pair combinations of particles produced in given event in some one large rapidity interval $\Delta \eta \in\left(Y_{1}, Y_{2}\right)$. The $B$ is the same, but in the case of uncorrelated particle production, obtained by the event mixing procedure.

In this definition in contrast with (3.9) one implies from the very beginning that the translation invariance in rapidity takes place and the result depends only on $\Delta \eta=\eta_{1}-\eta_{2}$ for any $\eta_{1}, \eta_{2} \in$ $\left(Y_{1}, Y_{2}\right)$. (All the pairs with the same value of difference $\eta_{1}-\eta_{2}$ contribute to the same bin of the multiplicity distribution, irrespective of the value of $\left(\eta_{1}+\eta_{2}\right) / 2$, see also the discussion in [16].) This assumption is reasonable only in the central rapidity region at high energies. It means that we suppose that in the interval $\left(Y_{1}, Y_{2}\right)$ :

$$
\rho_{1}(\eta)=\rho_{0}, \quad \rho_{2}\left(\eta_{1}, \eta_{2} ; \phi\right)=\rho_{2}\left(\eta_{1}-\eta_{2} ; \phi\right)
$$

(see formula (3.25)).

In this case we have for the enumerator in (6.1):

$$
S(\Delta \eta, \Delta \phi)=\int_{Y_{1}}^{Y_{2}} d \eta_{1} d \eta_{2} \rho_{2}\left(\eta_{1}-\eta_{2} ; \Delta \phi\right) \delta\left(\eta_{1}-\eta_{2}-\Delta \eta\right)
$$


or in the case of commonly used symmetric interval $(-Y / 2, Y / 2)$ :

$$
S(\Delta \eta, \Delta \phi)=\rho_{2}(\Delta \eta ; \Delta \phi) t_{Y}(\Delta \eta)
$$

where the $t_{Y}(\Delta \eta)$ is the "triangular" weight function (7.2), defined in Appendix A (see Fig.1).

In the denominator of (6.1) for mixed events we should replace the $\rho_{2}\left(\eta_{1}, \eta_{2} ; \Delta \phi\right)$ by the product $\rho_{1}\left(\eta_{1}\right) \rho_{1}\left(\eta_{2}\right)$, which due to the translation invariance in rapidity reduces simply to $\rho_{0}^{2}$. Then

$$
B(\Delta \eta, \Delta \phi)=\rho_{0}^{2} t_{Y}(\Delta \eta) .
$$

Substituting into (6.1) we get

$$
C(\Delta \eta, \Delta \phi)=\frac{\rho_{2}(\Delta \eta ; \Delta \phi)}{\rho_{0}^{2}}-1=C_{2}(\Delta \eta, \Delta \phi),
$$

where we have taken into account (3.9) and (3.25). We see that if the translation invariance in rapidity takes place within the interval $\left(Y_{1}, Y_{2}\right)$, then the definition (6.1) is equivalent to the standard one (3.9).

The drawback of this approach is that it supposes from the very beginning the translation invariance in rapidity and hence can't be applied for an experimental determination of the twoparticle correlation function $C_{2}$ for asymmetrical processes (such as e.g. the pA-interactions) and even for symmetric reactions at large rapidity distances, when the translation invariance (3.25) is not valid. At that by (3.24), (5.2) and (5.5) we see that the approaches based on the analysis of the standard (2.2) or modified (5.1), (5.4) FB correlation coefficients with two remote windows of small acceptance in rapidity and azimuth enable in any case to measure the correlation function $C_{2}\left(\eta_{1}, \eta_{2} ; \phi_{1}-\phi_{2}\right)$ without using of any event mixing procedure.

Note also that using of an event mixing procedure, applied in di-hadron correlation approach (6.1) for the determination of the $B$, can lead to an uncertainty in the experimental determination of the constant component of two-particle correlation function $C(\Delta \eta, \Delta \phi)$ even in central region, where the translation invariance in rapidity takes place and the definitions of the correlation functions $C(\Delta \eta, \Delta \phi)(6.1)$ and $C_{2}(\Delta \eta, \Delta \phi)$ (3.9) are equivalent to each other (6.7). Recall the importance of the experimental determination of the constant component of two-particle correlation function $C_{2}$, which corresponds to the contribution of the long-range (LR) correlations, (4.5), as it was mentioned in section 4 after the formulae (4.4) and (4.5).

The problem is that the two-particle correlation function $C(\Delta \eta, \Delta \phi)$, obtained by the di-hadron correlation approach (6.1), depends through $B$ on the details of the event mixing procedure, used for the imitation of uncorrelated particle production. Due to the uncertainties in the normalization factor $B$ one cannot measure correctly a value of the common pedestal, i.e. the long-range component of $C_{2}$.

The same effect also takes place if one uses some arbitrary, unjustified normalization procedures for the experimental determination of $S$ and/or $B$ in formula (6.1), e.g. the normalization of $S$ by the number of pairs produced in the given event, or the normalization of $B(\Delta \eta, \Delta \phi)$ by $B(0,0)$. As follows from the formulae (3.4)-(3.7), determining experimentally $C_{2}$ one should not introduce such additional normalization factors and has to take into account the contributions from different events at given $\Delta \eta, \Delta \phi$ with the same weight. 
Note that this long-range component of $C_{2}$ can be measured unambiguously in our approach (3.7), based on the studies of the FB correlations between multiplicities in windows separated in azimuth and rapidity, without using any event mixture procedure.

\section{Conclusions}

The connection between the FB multiplicity correlation coefficient $b$ in windows separated in azimuth and rapidity and the two-particle correlation function $C_{2}$ is traced. Basing on this connection the general features of the dependence of the correlation coefficient $b$ on the acceptances of the observation windows are established a model independent way.

We have compared the different definitions of the multiplicity correlation coefficient and see that the traditional definitions (2.2), (2.4) and (2.5) of the FB correlation coefficient lead to its strong dependence on the acceptance of the windows, with the correlation coefficient going to zero with the acceptance. Hence, the results obtained for the windows of different width can't be compared directly. In this connection we propose suitable observables (5.1) and (5.4) for the future FB correlation studies, which values have nonzero limit when the acceptance goes to zero.

We compare the FB correlation approach with the so-called di-hadron correlation analysis. We show a model independent way that the measurements of the FB correlation coefficient between multiplicities in two small windows separated in rapidity and azimuth enable to find the two-particle correlation function $C_{2}$ even when the particle distribution in rapidity is not flat, as e.g. in the case of pA interactions, and when the $C_{2}\left(\eta_{1}, \eta_{2} ; \Delta \phi\right)$ depends not only on the difference of rapidities, $\Delta \eta=\eta_{1}-\eta_{2}$, but both on $\eta_{1}$ and $\eta_{2}$. That is in the cases, when the traditional di-hadron correlation analysis, assuming from the very beginning the dependence of two-particle correlation function only on the differences $\Delta \eta$ and $\Delta \phi$, is not applicable.

We note that even in a mid-rapidity region, where the application of the di-hadron correlation approach is justified, the results obtained by this method depend on the details of track and/or event mixing used in the approach for the imitation of the uncorrelated particle production, what leads to the uncertainties in determination of the long-range correlation component (the common "pedestal") of $C_{2},(4.5)$, which value contains the important physical information on the magnitude of the event-by-event fluctuation in the number of sources.

Important that this long-range component of $C_{2}$ can be measured unambiguously, by the FB correlation approach, (3.7), which does not use any event mixture procedure, and hence avoids the associated uncertainties.

\section{Appendix A. Calculation of the integrals over rapidity and azimuth windows.}

For symmetric rapidity windows $\delta \eta_{B}=\delta \eta_{F}=\delta \eta$ with the distance $\eta_{F B}=\eta_{F}-\eta_{B}$ between their centers one has

$$
\int_{\delta \eta_{F}} d \eta_{1} \int_{\delta \eta_{B}} d \eta_{2} f\left(\left|\eta_{1}-\eta_{2}\right|\right)=\int_{-\delta \eta}^{\delta \eta} d \eta f\left(\left|\eta_{F B}+\eta\right|\right) t_{\delta \eta}(\eta)
$$

where $t_{\delta y}(y)$ is the "triangular" weight function (see Fig.1):

$$
t_{\delta y}(y)=[\theta(-y)(\delta y+y)+\theta(y)(\delta y-y)] \theta(\delta y-|y|) .
$$




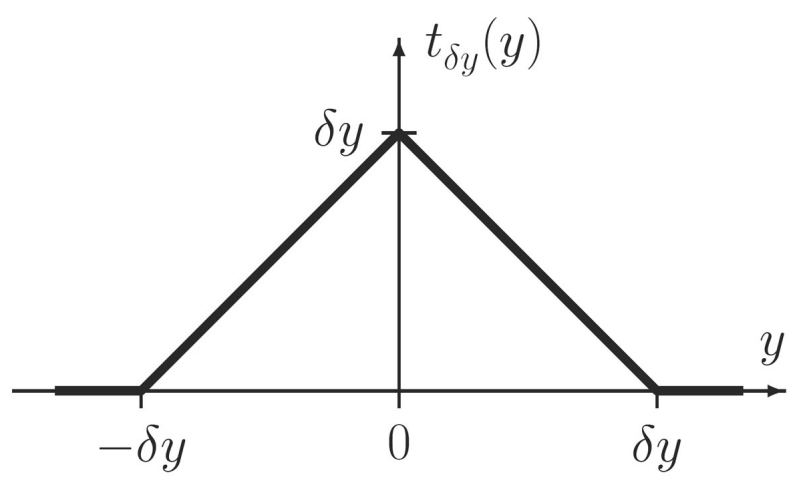

Figure 1: The "triangular" weight function arising due to phase space at integration over non-periodical FB windows (see Appendix A for details).

The formula (7.1) is valid for any distance between the centers of windows, in particular for coinciding windows. In the last case $\eta_{F B}=0$ and we have

$$
\int_{\delta \eta_{F}} d \eta_{1} \int_{\delta \eta_{F}} d \eta_{2} f\left(\left|\eta_{1}-\eta_{2}\right|\right)=\int_{-\delta \eta}^{\delta \eta} d \eta f(|\eta|) t_{\delta \eta}(\eta)=2 \int_{0}^{\delta \eta} d \eta f(|\eta|)(\delta \eta-\eta)
$$

The same formula

$$
\int_{\delta \phi_{F}} d \phi_{1} \int_{\delta \phi_{B}} d \phi_{2} f\left(\left|\phi_{1}-\phi_{2}\right|\right)=\int_{-\delta \phi}^{\delta \phi} d \phi f\left(\left|\phi_{F B}+\phi\right|\right) t_{\delta \phi}(\phi)
$$

is valid for the integration over azimuthal windows, but in this case one has also to take into account the periodicity: $f(|\phi|)=f(|\phi+2 \pi k|)$. The last leads to significant simplification of the formula (7.4) in the case of full, $2 \pi$, azimuth acceptance windows:

$$
\int_{-2 \pi}^{2 \pi} d \phi f\left(\left|\phi_{F B}+\phi\right|\right) t_{2 \pi}(\phi)=4 \pi \int_{0}^{\pi} d \phi f(|\phi|)
$$

So for large symmetric windows in the central rapidity region by (7.1)-(7.3) the formulae (3.29) and (3.30) in general case can be written in the following form

$$
\begin{gathered}
I_{F B}=(\delta \eta \delta \phi)^{-2} \int_{-\delta \eta}^{\delta \eta} d \eta \int_{-\delta \phi}^{\delta \phi} d \phi t_{\delta \eta}(\eta) t_{\delta \phi}(\phi) C_{2}\left(\eta_{F B}+\eta, \phi_{F B}+\phi\right) \\
I_{F F}=4(\delta \eta \delta \phi)^{-2} \int_{0}^{\delta \eta} d \eta \int_{0}^{\delta \phi} d \phi(\delta \eta-y)(\delta \phi-\phi) C_{2}(\eta, \phi) .
\end{gathered}
$$

The $\delta \eta$ and $\delta \phi$ are the width of the observation windows in rapidity and in azimuth, and the $\eta_{F B}$ and $\phi_{F B}$ are the corresponding distances between their centers. We imply that $C_{2}(\eta, \phi)$ satisfies the following conditions

$$
C_{2}(-\eta, \phi)=C_{2}(\eta, \phi), \quad C_{2}(\eta,-\phi)=C_{2}(\eta, \phi), \quad C_{2}(\eta, \phi+2 \pi k)=C_{2}(\eta, \phi) .
$$




\section{References}

[1] S. Uhlig, I. Derado, R. Meinke, and H. Preissner, Observation of charged particle correlations between the forward and backward hemispheres in pp collisions at ISR energies, Nucl. Phys. B 132 (1978) 15.

[2] K. Alpgard et al. (UA5 Collaboration), Forward-backward multiplicity correlations in p anti-p collisions at $540 \mathrm{GeV}$, Phys. Lett. B 123 (1983) 361.

[3] R.E. Ansorge et al. (UA5 Collaboration), Charged particle correlations in p̄p collisions at c.m. energies of $200 \mathrm{GeV}, 546 \mathrm{GeV}$ and $900 \mathrm{GeV}$, Z. Phys. C 37 (1988) 191.

[4] T. Alexopoulos et al. (E735 Collaboration), Charged particle multiplicity correlations in p $\bar{p}$ collisions at $\sqrt{s}=0.3 \mathrm{TeV}$ to $1.8 \mathrm{TeV}$, Phys. Lett. B 353 (1995) 155.

[5] B.I. Abelev et al. (STAR Collaboration), Growth of long range forward-backward multiplicity correlations with centrality in AuAu collisions at $\sqrt{s}=200 \mathrm{GeV}$, Phys. Rev. Lett. 103 (2009) 172301 [arXiv:0905.0237, nucl-ex].

[6] G. Aad et al. (ATLAS Collaboration), Forward-backward correlations and charged-particle azimuthal distributions in pp interactions using the ATLAS detector, JHEP 1207 (2012) 019 [arXiv:1203.3100, hep-ex].

[7] A. Capella and A. Krzywicki, Unitarity corrections to short range order: long range rapidity correlations, Phys. Rev. D 18 (1978) 4120.

[8] N.S. Amelin et al., Long and short range correlations and the search of the quark gluon plasma, Phys. Rev. Lett. 73 (1994) 2813.

[9] M.A. Braun, C. Pajares and V.V. Vechernin, On the forward-backward correlations in a two stage scenario, Phys. Lett. B 493 (2000) 54 [arXiv: hep-ph/ 0007241$].$

[10] M.A. Braun, R.S. Kolevatov, C. Pajares and V.V. Vechernin, Correlations between multiplicities and average transverse momentum in the percolating color strings approach, Eur. Phys. J. C 32 (2004) 535 [arXiv: hep-ph/0307056].

[11] P. Brogueira, J. Dias de Deus and J.G. Milhano, Forward-backward rapidity correlations at all rapidities, Phys. Rev. C 76 (2007) 064901 [arXiv: 0709.3913 , hep-ph].

[12] N. Armesto, L. McLerran and C. Pajares, Long range forward-backward correlations and the color glass condensate, Nucl. Phys. A 781 (2007) 201 [arXiv: hep-ph/0607345].

[13] V.V. Vechernin and R.S. Kolevatov, On multiplicity and transverse-momentum correlations in collisions of ultrarelativistic ions, Phys. Atom. Nucl. 70 (2007) 1797.

[14] V.P. Konchakovski et al., Forward-backward correlations in nucleus-nucleus collisions: Baseline contributions from geometrical fluctuations, Phys. Rev. C 79 (2009) 034910 [arXiv:0812.3967, nucl-th].

[15] T. Lappi and L. McLerran, Long range rapidity correlations as seen in the STAR experiment, Nucl. Phys. A 832 (2010) 330 [arXiv:0909.0428, hep-ph].

[16] A. Bzdak, Symmetric correlations as seen in central AuAu collisions at $\sqrt{s}=200 \mathrm{~A}$ GeV, Phys. Rev. C 85 (2012) 051901 [arXiv:1108.0882, hep-ph].

[17] A. Olszewski, W. Broniowski, Forward-backward multiplicity correlations in relativistic heavy-ion collisions in a superposition approach, Phys. Rev. C 88 (2013) 044913 [arXiv: 1303.5280 , nucl-th]. 
[18] V.V. Vechernin, R.S. Kolevatov, Simple cellular model of long-range multiplicity and p correlations in high-energy nuclear collisions, Vestnik SPbU ser.4, no.2 (2004) 12 [arXiv: hep-ph/0304295].

[19] V.V. Vechernin, Long-range rapidity correlations in the model with independent emitters, in Proceedings of the Baldin ISHEPP XX vol.2, JINR, Dubna (2011) 10 [arXiv: 1012.0214 , hep-ph].

[20] ALICE collaboration et al., ALICE: Physics Performance Report Volume II, J. Phys. G 32 (2006) 1295 [Section: 6.5.15 - Long-range correlations, p.1749]

[21] C. Pruneau, S. Gavin, and S. Voloshin, Methods for the study of particle production fluctuations, Phys. Rev. C 66 (2002) 044904 [arXiv:nucl-ex/ 0204011 ].

[22] L. Foa, Inclusive study of high-energy multiparticle production and two-body correlations, Phys. Rep. 22 (1975) 1

[23] J. Whitmore, Multiparticle production in the Fermilab bubble chambers, Phys. Rep. 27 (1976) 187

[24] B.I. Abelev et al. (STAR Collaboration), Long range rapidity correlations and jet production in high energy nuclear collisions, Phys. Rev. C 80 (2009) 064912 [arXiv:0909.0191, nucl-ex].

[25] CMS Collaboration, Observation of long-range near-side angular correlations in proton-proton collisions at the LHC, JHEP 1009 (2010) 091 [arXiv:1009.4122, hep-ex].

[26] V.V. Vechernin and H.S. Nguyen, Fluctuations of the number of participants and binary collisions in AA interactions at fixed centrality in the Glauber approach, Phys. Rev. C 84 (2011) 054909 [arXiv:1102.2582, hep-ph].

[27] G.A. Feofilov, et al. (for ALICE collaboration), Forward-backward multiplicity correlations in pp collisions in ALICE at 0.9, 2.76 and $7 \mathrm{TeV}$, in proceedings of XXI International Baldin Seminar on High Energy Physics Problems POS (Baldin ISHEPP XXI) 075. 